\title{
Pneumatic Pulsatile Ventricular Assist Device as a Bridge to Heart Transplantation in Pediatric Patients
}

\author{
Antonio Amodeo, Gianluca Brancaccio, Guido Michielon, Sergio Filippelli, Zaccaria Ricci, \\ Stefano Morelli, Maria Giulia Gagliardi, Roberta Iacobelli, Giacomo Pongiglione, \\ and Roberto M. Di Donato
}

Department of Cardiac Surgery, Ospedale Pediatrico Bambino Gesù, Rome, Italy

\begin{abstract}
Despite the remarkable advances with the use of ventricular assist devices (VAD) in adults, pneumatic pulsatile support in children is still limited. We report on our experience in the pediatric population. A retrospective review of 17 consecutive children offered mechanical support with Berlin Heart as a bridge to heart transplant from February 2002 to April 2010 was conducted. The median patient age was 3.9 years ( 75 days to 13.3 years). The median patient weight was $14.1 \mathrm{~kg}(2.9-43 \mathrm{~kg})$. Before VAD implantation, all children were managed by multiple intravenous inotropes and mechanical ventilation (14) or extracorporeal membrane oxygenation (3). All patients had right ventricular dysfunction. Nine patients required biventricular mechanical support (BVAD), but in all other cases a single left ventricular assist device proved sufficient (47\%). The median duration of VAD support was 47 days (1-168 days). The median pre-VAD pulmonary vascular resistance index (Rpi) was $5.7 \mathrm{WU} / \mathrm{m}^{2}$ (3.5 to $\left.14.4 \mathrm{WU} / \mathrm{m}^{2}\right)$. Eleven patients $(65 \%)$ were successfully bridged to heart transplantation after a median duration of mechanical support of 68 days (6-168 days). Six deaths occurred $(35 \%)$, three for neurological complications, one for sepsis,
\end{abstract}

and two others for device malfunctioning. Since 2007, the survival rate of our patients has increased from $43 \%$ to $80 \%$, and the need for BVAD has decreased from $86 \%$ to $30 \%$. In two patients with Rpi $>10 \mathrm{WU} / \mathrm{m}^{2}$, unresponsive to pulmonary vasodilatator therapy, Rpi dropped to 2.2 and $2 \mathrm{WU} / \mathrm{m}^{2}$ after 40 and 23 days of BVAD support, respectively. Seven patients $(41 \%)$ required at least one pump change. Of 11 patients undergoing heart transplant, four developed an extremely elevated $(>60 \%)$ panel reactive antibody by enzyme-linked immunosorbent assay, confirmed by Luminex. All of them experienced at least one acute episode of rejection in the first month after heart transplant, needing plasmapheresis. The survival rate after heart transplantation was $100 \%$ with a median follow-up of 25.4 months (6 days to 7.7 years). Mechanical support in children with end-stage heart failure is an effective strategy as a bridge to heart transplantation with a reasonable morbidity and mortality. BVAD support may offer an additional means to reverse extremely elevated pulmonary vascular resistance. Key Words: Circulatory assist devices-Infant-Heart transplantation-Pulsatile-Ventricular assist device.
Although considerable progress has been made with ventricular assist devices (VADs) for adults, pneumatic pulsatile circulatory support in children is still limited (1-3). doi:10.1111/j.1525-1594.2010.01144.x

Received July 2010; revised August 2010.

Address correspondence and reprint requests to Dr. Gianluca Brancaccio, Department of Cardiac Surgery, Ospedale Pediatrico Bambino Gesù, Piazza S.Onofrio,5- 00165 Rome, Italy. E-mail: gbrancaccio70@hotmail.com

Presented in part at the 6th International Conference on Pediatric Mechanical Circulatory Support Systems and Pediatric Cardiopulmonary Perfusion held May 6-8, 2010 in Boston, MA, USA.
In common practice, non-pulsatile devices, for example, extracorporeal membrane oxygenation (ECMO) and centrifugal pumps, have been the mainstay of pediatric circulatory support strategy (4). These technologies provide immediate cardiopulmonary support; however, their long-term use is associated with considerable risks (5). On the other side, some pulsatile VADs have evolved into effective long-term bridge measures toward heart transplantation. However, thus far they have been available only for adults or adolescents $(1,6-8)$. With the miniaturized Berlin Heart EXCOR (Berlin Heart, Berlin Heart AG, Berlin, Germany), there is now a VAD conceived for long-term mechanical circulatory support in newborns and young infants. 
The aim of this study is to present the series of pediatric implants of Berlin Heart EXCOR at Bambino Gesù Children's Hospital.

\section{PATIENTS AND METHODS}

\section{Patients}

This is a retrospective, nonrandomized review of 17 consecutive patients who underwent insertion of a Berlin Heart EXCOR at Bambino Gesù Children's Hospital between February 2002 and April 2010.

After parental consent, all medical records were reviewed. Data points are summarized in tabular format and expressed as a mean or median value with observed extremes where appropriate. The study was approved by the Medical Advisory Board of the hospital.

\section{The Berlin Heart EXCOR}

The Berlin Heart consists of a paracorporeal, pneumatically driven, polyurethane blood pump (10-2530-50-60 mL) with a multilayer flexible membrane separating the blood from the air chamber. Silicon cannulae connect the blood pump to the patient, and tri-leaflet inflow and outflow valves prevent blood reflux. All surfaces in contact with blood are heparincoated. Each pump is driven by a pulsatile electropneumatic system. The drive unit (IKUS 2000, Berlin Heart) has a triple operational control and pneumatic system; synchronous and asynchronous operating models are available.

\section{Surgical procedure}

The operations were performed through a standard median sternotomy, using mildly hypothermic cardiopulmonary bypass. Before 2007, the cannulae for the left ventricular assist device (LVAD) were inserted under aortic cross-clamping, and the site for inflow cannulation was the left atrium. Since August 2007, following the indications of the German Heart Center, we changed the technique and site of cannulae insertion. The heart was kept beating throughout the procedure, except for variable periods of induced ventricular fibrillation for inflow cannulations. Inflow cannulation for LVADs was preferentially achieved via the left ventricular apex, except in selected cases. Left-sided outflow cannulation was into the proximal ascending aorta. Right atrial to main pulmonary artery cannulation was used for the right side of the heart. When a biventricular mechanical support (BVAD) was employed, it was run in a synchronous operating mode. In the last patient of the series, concomitant closure of an atrial communication was accomplished.
All patients included in the study were monitored by near-infrared spectroscopy (NIRS) during surgical procedures.

\section{Anticoagulation}

Postoperative anticoagulation was started with intravenous heparin and dipyridamole $(10 \mathrm{mg} / \mathrm{kg} /$ day) $24 \mathrm{~h}$ after admission to the intensive care unit (ICU). Heparin infusion was titrated, four times a day, to adjust the partial thromboplastin time (PTTs) between 40 and $60 \mathrm{~s}$. Antithrombin III (ATIII) was corrected to achieve values higher than $100 \%$. The chronic anticoagulation regimen was initiated on postoperative day 3 with oral warfarin to keep the international normalized ratio (INR) between 3 and 3.5. INR was monitored once a day since a normal value (3-3.5) was reached. Aspirin $(5 \mathrm{mg} / \mathrm{kg} /$ dose p.o. q.i.d.) was added in accordance with thromboelastogram indications.

\section{Statistical analysis}

Statistical analysis was conducted with the SASStatview-1998 statistical software, SAS Institute, Inc., Cary, NC. Continuous variables were expressed as mean and range. Categorical analysis was conducted by chi-square and Fisher's exact test. Freedom from time-related events (death) was conducted according to actuarial and/or Kaplan-Meier technique; the resulting curves with $95 \%$ confidence limits were compared with log-rank testing, and nomograms of the hazard function were obtained.

\section{RESULTS}

\section{Preoperative data}

Patient characteristics and demographics are summarized in Table 1 . The study included 12 boys and 5 girls, with a mean age of 3.9 years ( 75 days to 13.3 years) and a mean weight of $14.1 \mathrm{~kg}(2.9-43 \mathrm{~kg})$. The etiology of heart failure was dilative cardiomyopathy in 14 (secondary to myocarditis in two), restrictive cardiomyopathy in two, and postcardiotomy cardiac dysfunction in one.

\section{Preoperative status}

Hemodynamic support was managed by milrinone $1 \mu \mathrm{g} / \mathrm{kg} / \mathrm{min}$, dopamine $(5-10 \mu \mathrm{g} / \mathrm{kg} / \mathrm{min})$ and epinephrine $0.01-0.1 \mu \mathrm{g} / \mathrm{kg} / \mathrm{min}$ (median inotropic score $25 \pm 3$ ). Fourteen patients were receiving mechanical ventilation at the time of VAD implantation and three patients were on ECMO for cardiorespiratory failure or failed weaning off cardiopulmonary bypass. All had severe systemic ventricular dysfunction, with a median ejection fraction of 
TABLE 1. Summary of patient demographics, mechanical support data, and outcome

\begin{tabular}{|c|c|c|c|c|c|c|c|c|}
\hline Patients & Age (months) & Sex & Weight (kg) & Diagnosis & Support (days) & LVAD/BVAD & Complication & Outcome \\
\hline \multicolumn{9}{|c|}{ Before 2007} \\
\hline 1 & 1.2 & $\mathrm{~F}$ & 2.9 & post-CEC & 2 & LVAD & Cer.Isch. & Death \\
\hline 2 & 20.6 & $\mathrm{M}$ & 10 & CMPD & 20 & BVAD & Cer.Hem. & Death \\
\hline 3 & 46 & $\mathrm{M}$ & 15 & CMPD & 16 & BVAD & - & Transplant \\
\hline 4 & 89.1 & $\mathrm{M}$ & 22 & SVA & 1 & BVAD & Dev.Malf. & Death \\
\hline 5 & 115.2 & $\mathrm{M}$ & 27 & CMPD & 9 & BVAD & - & Transplant \\
\hline 6 & 58.3 & $\mathrm{~F}$ & 13 & CMPR & 72 & BVAD & - & Transplant \\
\hline 7 & 65.1 & $\mathrm{M}$ & 20 & CMPD & 22 & BVAD & Dev.Malf. & Death \\
\hline \multicolumn{9}{|c|}{ After 2007} \\
\hline 8 & 10.8 & $\mathrm{M}$ & 6.3 & CMPD & 54 & LVAD & Sepsis & Death \\
\hline 9 & 26.4 & $\mathrm{M}$ & 9.6 & CMPD & 40 & BVAD & Rejection & Transplant \\
\hline 10 & 5.7 & $\mathrm{M}$ & 5 & CMPD & 131 & LVAD & Cer.Hem./Rej. & Transplant \\
\hline 11 & 11.3 & $\mathrm{M}$ & 8.4 & CMPD-M & 69 & LVAD & - & Transplant \\
\hline 12 & 3.6 & $\mathrm{M}$ & 4 & CMPD & 37 & LVAD & Rej. & Transplant \\
\hline 13 & 2.5 & M & 4.5 & CMPR & 13 & BVAD & Cer.Isch. & Death \\
\hline 14 & 7.7 & $\mathrm{~F}$ & 7.5 & CMPD-M & 168 & LVAD & Sepsis & Transplant \\
\hline 15 & 7.2 & $\mathrm{~F}$ & 5.8 & CMPD & 108 & LVAD & - & Transplant \\
\hline 16 & 160.1 & $\mathrm{~F}$ & 43 & CMPD & 6 & BVAD & - & Transplant \\
\hline 17 & 152 & $\mathrm{M}$ & 35 & CMPD & 35 & LVAD & - & Transplant \\
\hline
\end{tabular}

BVAD, biventricular assist device; CEC, extracorporeal circulation; Cer.Isch., cerebral ischemia; CMPD, dilated cardiomyopathy; CMPD-M, dilated cardiomyopathy post-myocarditis; CMPR, restrictive cardiomyopathy; Dev. Malf., device malfunctioning; F, female; Cer.Hem., cerebral hemorrhage; LVAD, left ventricular assist device; M, male; MOF, multi-organ failure; Rej., myocardial rejection; SVA, aortic valve stenosis.

$28 \pm 2 \%$ (range from $18 \%$ to $30 \%$ ). No patient had signs of severe renal dysfunction before surgical procedure or required dialysis. The median pre-VAD pulmonary vascular resistance index (Rpi) was $5.7 \mathrm{WU} / \mathrm{m}^{2}\left(3.5-14.4 \mathrm{WU} / \mathrm{m}^{2}\right)$. At the time of Berlin Heart implantation, two patients (patients 6 and 9) presented with a total pulmonary vascular resistance $>10 \mathrm{WU} / \mathrm{m}^{2}$ unresponsive to pulmonary vasodilator therapy. After 72 and 40 days of BVAD assistance, Rpi dropped to $2.2 \mathrm{WU} / \mathrm{m}^{2}$ and $2 \mathrm{WU} / \mathrm{m}$, respectively, allowing the patients to be reconsidered for heart transplantation.

\section{VAD implantation}

Inflow cannulation for the LVAD was achieved via the left ventricular apex in 10 children and via the left atrium in seven children. The left ventricular apical cannulation (Fig. 1) was associated with higher survival $(P=0.0053)$. From August 2007, we used atrial cannulation only in selected cases of restrictive cardiomyopathy (patient 13), in which extensive myectomy is unsafe.

\section{Mechanical support management}

An isolated LVAD was used in eight patients and a BVAD in nine (Table 1), but since 2007 the need for BVAD has decreased to about $30 \%$. Use of LVAD $(P=0.18)$ was associated with a higher probability of survival (Fig. 2). The median duration of VAD support was 47 days (1-168 days). No patient required dialysis postoperatively. In the last 11 patients, inhaled nitric oxide $20 \mathrm{ppm}$ was started immediately after the surgical procedure. As soon as bowel motility allowed enteral administration, the patients were started on sildenafil via nasogastric tube $(0.5 \mathrm{mg} / \mathrm{kg}$ every $6 \mathrm{~h})$. No significant results were collected using the NIRS system.

\section{Outcomes}

On the whole, 11 patients (65\%) successfully endured the period of circulatory support, but since 2007 , eight of 10 patients $(80 \%)$ have been successfully bridged to heart transplant. As shown in Fig. 3 the year of implant $(P=0.025)$ influences the survival.

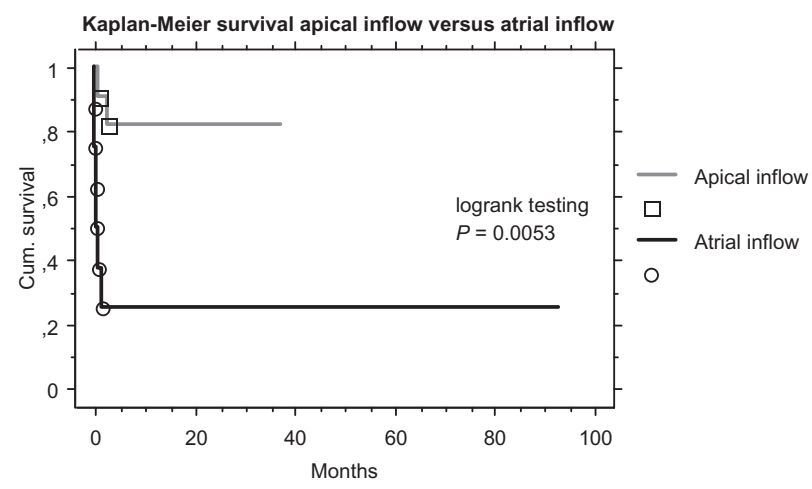

FIG. 1. Kaplan-Meier survival probability according to the type of site of cannulation. 
Kaplan-Meier survival LVAD versus BVAD

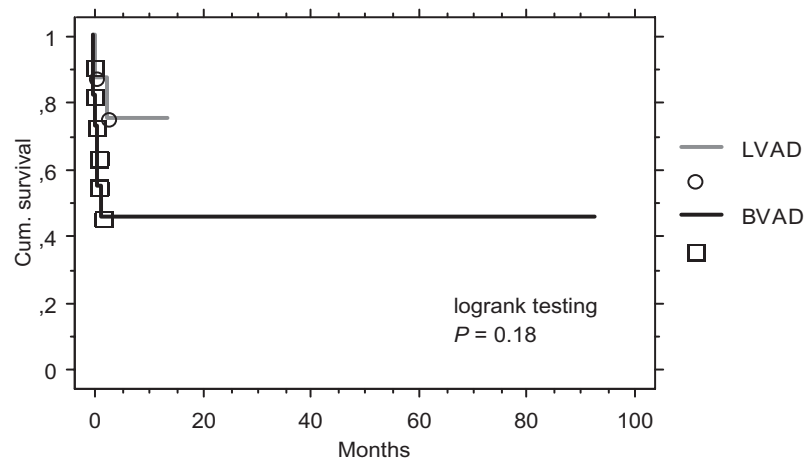

FIG. 2. Kaplan-Meier survival probability according to type of VAD.

There were six deaths, four before 2007, and two after 2007. Patient 1 suffered from a myocardial infarction for coronary artery injury during surgical correction of a supra-valvular mitral valve ring and required ECMO support. Later, he was switched to LVAD as a bridge to heart transplantation, but died on postoperative day 2 due to neurologic complications. Patient 2 underwent an elective BVAD implantation for dilative cardiomyopathy and, on postoperative day 18 , experienced a cerebral hemorrhage with brain death on postoperative day 20. Patients 4 and 7 had complications related to device malfunctioning, with acute low cardiac output in patient 4 and progressive cardiac dysfunction in patient 7 , respectively.

Patient 8 suffered from device infection with endocarditis and sepsis. After 54 days, he underwent an emergency removal of the device, showing a partial recovery of the left ventricular function; however, he died a week later due to sepsis and congestive heart failure. Another child with severe congestive heart failure and pulmonary edema (patient

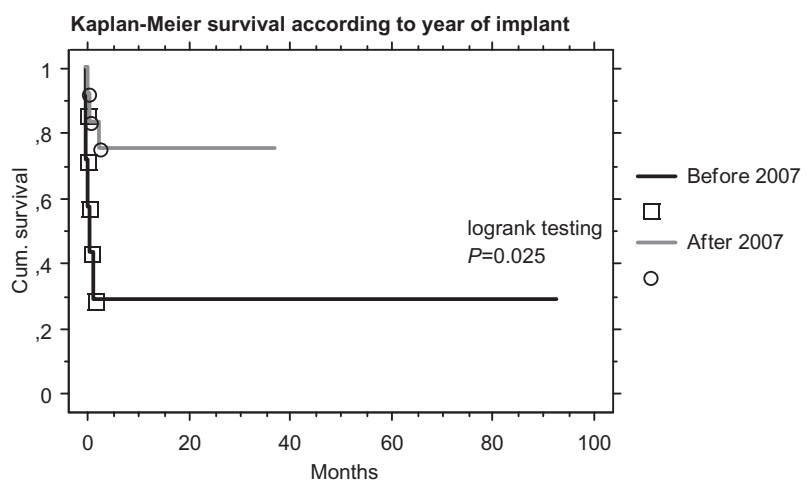

FIG. 3. Kaplan-Meier survival probability according to year of implant (before and after 2007).
TABLE 2. Adverse events during VAD support

\begin{tabular}{lc}
\hline Adverse event & No. of patients \\
\hline Pump change & $7 / 17(41 \%)$ \\
Infection & $8 / 17(47 \%)$ \\
Respiratory & $4 / 17(23 \%)$ \\
Driveline & $2 / 17(12 \%)$ \\
Sepsis & $4 / 17(23 \%)$ \\
Neurologic complications & $4 / 17(23 \%)$ \\
Ischemic & $2 / 17(12 \%)$ \\
Hemorrhagic & $2 / 17(12 \%)$ \\
Prolonged ventilator dependence & $4 / 17(23 \%)$ \\
Percutaneous closure of PFO/ASD & $2 / 17(12 \%)$ \\
Death & $6 / 17(35 \%)$ \\
\hline
\end{tabular}

ASD, atrial septal defect; PFO, patent foramen ovalis.

13) underwent BVAD implantation with consequent lung recovery. On postoperative day 7, a thrombus materialized in the left pneumatic ventricle, requiring a pump change. This procedure was complicated by massive air embolism with severe cerebral ischemia. The patient died after 13 days of support of pulmonary and cerebral complications.

Adverse events during VAD support are summarized in Table 2. Patient 10 had a cerebral hemorrhage with secondary hydrocephalus, necessitating a ventriculo-peritoneal drainage. Rather unexpectedly, after 1 month of cerebral decompression, clinical recovery was observed and the patient was re-enrolled for heart transplant. After 6 months of heart transplant he is well, without neurologic complications. Patients 11 and 12, a few hours after Berlin Heart implantation, developed a severe arterial oxygen desaturation $(<65 \%)$ secondary to right-toleft shunting due to an unrecognized patent foramen ovale/atrial septal defect. The patients underwent a percutaneous closure of the atrial communication by an Amplatzer device (AGA Medical Corporation, Golden Valley, MN, USA) on postoperative day 1. A total of seven pump replacements were needed in six different patients, consequent to fibrin deposition or thombus. In patients 6 and 9, after a median follow-up of 28 months of heart transplant, Rpi remained normal without pulmonary vasodilatators.

\section{Follow-up}

Overall, 11 of 17 patients were successfully bridged to heart transplant. Post-transplant survival has been $100 \%$, with a median follow-up of 25.4 months (6 days to 7.7 years).

\section{Sensitization}

Human leukocyte antigen sensitization, calculated as dithiothreitol-treated T-cell panel-reactive antibody titer, was less than $10 \%$ immediately before 
device implantation and heart transplantation in all patients. However, four children experienced an extremely elevated titer after heart transplant (>60\%). In all patients, the diagnosis by enzyme-linked immunosorbent assay (ELISA) was confirmed by Luminex, and patient 14 had a positive donor-specific B-cell crossmatch. Each patient, except one with a systemic immunologic disorder, had been mechanically supported longer than 30 days and experienced at least one episode of acute rejection in the first month after heart transplant, needing plasmapheresis.

\section{DISCUSSION}

Mechanical support as a bridge to heart transplantation improves survival in children with end-stage heart failure $(9,10)$. ECMO remains the most common type of mechanical support available at pediatric age, especially in acute forms of heart failure, for example, in the cases of fulminant myocarditis or failed weaning off cardiopulmonary bypass (10-12). ECMO assures total cardiopulmonary support and allows a flexibile choice between peripheral and central cannulation. However, it provides nonpulsatile flow, restricts patient mobility, and presents a high incidence of hemorrhagic and infective complications in the long-term use (5).

The use of pulsatile, paracorporeal, closed-circuit VADs significantly extends the support time, influencing the strategy in younger children. Pulsatile pumping results in better tissue perfusion and a more extensive recruitment of the microcirculation of the brain, lungs, and kidneys. In addition, by reversing end-organ dysfunction, pulsatile VADs improve the overall clinical condition and the likelihood of a successful heart transplantation (3,13-15).

Our institutional experience with Berlin Heart began in 1999, but until 2007 we used a left atrium for left-sided inflow cannulation with high incidence of severe neurologic complications, leading to a hospital mortality of $57 \%$. From 2007, we switched to preferential left ventricular apex cannulation, as suggested by the German Heart Center in Berlin, and the ensuing survival rate of $80 \%$ now compares favorably with other series. Studies examining pediatric bridge to transplant experiences have reported bridging success rates between $51 \%$ and $89 \%$ and 1 -year posttransplant survival rates ranging from $62 \%$ to $100 \%$ $(7,10,13,15-17)$. Our post-transplant survival after VAD support has been excellent, with $100 \%$ of children alive to date.

Another advantage of the apical cannulation is the less frequent need for an additional mechanical right heart support ( $86 \%$ before 2007 vs. $30 \%$ after 2007).
Adequate unloading of the left ventricle reduces the left ventricular end-diastolic pressure and, in turn, acutely reduces the afterload of the right ventricle (3). So, our strategy is to first implant an LVAD and intraoperatively manage pulmonary vascular resistance by selective pharmacologic agents (nitric oxide, oxygen, hyloprost, and a small amount of catecholamines). At subsequent evaluation of right ventricular function by trans-esophageal echocardiography, often we find that there is no need for an additional mechanical right heart support. This lowers the risk of long-term complications, even if some reports showed few disadvantages with routine implantation of BVADs (7).

\section{Timing}

Timing of device implantation is of critical importance. We followed the suggestions of the German Heart Center $(16,18)$. The goal is to avoid premature exposure to mechanical support and device-related morbidities, but at the same time, to prevent the onset of any end-organ dysfunction. Our indications for VAD implantation in a child with end-stage heart failure now include the need for more than two inotropes, need for mechanical ventilation with at least one failed attempt at weaning, and need for ECMO without any evidence of ventricular recovery.

The method of mechanical assistance is chosen according to the presumed likelihood of, and length of time for, ventricular functional recovery. All children needing mechanical assistance undergo a myocardial biopsy. If the diagnosis is acute myocarditis, ECMO is the preferred choice, for a high chance of ventricular recovery during a short-term period. Otherwise, a long-term assistance method, for example, by Berlin Heart, is preferred.

Our results confirm the notion that patients with end-stage heart disease and severe pulmonary hypertension may become good candidates for heart transplantation after a more or less prolonged BVAD support $(19,20)$. In two patients with elevated pulmonary vascular resistance, the Rpi normalized after BVAD support and remained normal after heart transplant.

The theoretical basis for ventricular assist device implantation in similar cases is that continuous unloading of the left ventricle, provided by the LVAD, lessens the left atrial pressure and that the antegrade blood driven by the right VAD promotes, at the same time, the decline of pulmonary vascular resistance. This theory was substantiated in adult patients (20).

\section{Complications}

In this series, $23 \%$ of patients suffered from neurologic events. A similar neurologic morbidity rate was 
noted by Cassidy et al. and by the Stanford group $(8,13)$. Most likely, neurologic complications are secondary to left atrial cannulation, as reported in the literature. This type of complications and the rather elevated number of necessary pump changes (41\%), especially in low-weight patients, also suggest that the protocol for anticoagulation remains a major issue in the management of these patients.

Almost half of our patients had infective complications and in one case infection was the cause of death. The improvements observed in cumulative survival and in reduction of infections since 2007 are secondary to improvements in surgical technique and to changes in ICU management. In fact, we currently attempt to close the sternum whenever possible (33\% before 2007 vs. $91 \%$ after 2007). Furthermore, we have changed the antimicrobial prophylaxis resorting to a broad spectrum coverage, including vancomycin (continuous intravenous infusion), meropenem, and amikacin.

Almost one-third of our patients undergoing heart transplantation after mechanical support (36\%) showed evidence of sensitization by ELISA, confirmed by Luminex methodology. Two-thirds of them were low-weight $(<10 \mathrm{~kg})$ infants, and experienced one episode of acute rejection in the first month after transplant. The cause of this occurrence remains mostly unknown, but it is alleged to prolong waiting time to transplantation, increase risk of acute rejection, and increase 30-day mortality rate after heart transplantation (21).

\section{Limitations}

This study is limited by its retrospective, nonrandomized nature. Furthermore, the small number of patients imposes a careful interpretation of data analysis, which, undoubtedly, requires further confirmation.

\section{CONCLUSIONS}

Insertion of pulsatile paracorporeal VADs in children with end-stage heart failure is an effective strategy as a bridge to heart transplantation. This study demonstrates the feasibility and the relative safety of VAD support in the pediatric population, and confirms that BVAD support may offer an additional means to reverse extremely elevated pulmonary vascular resistance.

\section{REFERENCES}

1. Rose EA, Gelijns AC, Moskowitz AJ, et al. Long-term mechanical left ventricular assistance for end-stage heart failure. N Engl J Med 2001;345:1435-43.
2. Hetzer R, Loebe M, Potapov EV, et al. Circulatory support with pneumatic paracorporeal ventricular assist device in infants and children. Ann Thorac Surg 1998;66:1498-506.

3. Stiller B, Weng Y, Hubler M, et al. Pneumatic pulsatile ventricular assist devices in children under 1 year of age. Eur J Cardiothorac Surg 2005;28:234-9.

4. Chang AC, McKenzie ED. Mechanical cardiopulmonary support in children and young adults: extracorporeal membrane oxygenation, ventricular assist devices, and long-term support devices. Pediatr Cardiol 2005;26:2-28.

5. Ibrahim AE, Duncan BW, Blume ED, Jonas RA. Long-term follow-up of pediatric cardiac patients requiring mechanical circulatory support. Ann Thorac Surg 2000;69:186-92.

6. Reinhartz O, Stiller B, Eilers R, Farrar DJ. Current clinical status of pulsatile pediatric circulatory support. ASAIO J 2002:48:455-9.

7. Gandhi SK, Huddleston CB, Balzer DT, Epstein DJ, Boschert TA, Canter CE. Biventricular assist devices as a bridge to heart transplantation in small children. Circulation 2008;118:S89S93.

8. Malaisrie SC, Pelletier MP, Yun JJ, et al. Pneumatic paracorporeal ventricular assist device in infants and children: initial Stanford experience. J Heart Lung Transplant 2008;27:173-7.

9. Garcia-Guereta L, Cabo J, de La Oliva P, et al. Ventricular assist device application with the intermediate use of a membrane oxygenator as a bridge to pediatric heart transplantation. J Heart Lung Transplant 2009;28:740-2.

10. Goldman AP, Cassidy J, de Leval M, et al. The waiting game: bridging to paediatric heart transplantation. Lancet 2003;362:1967-70.

11. del Nido PJ, Armitage JM, Fricker FJ, et al. Extracorporeal membrane oxygenation support as a bridge to pediatric heart transplantation. Circulation 1994.

12. Pollock JC, Charlton MC, Williams WG, Edmonds JF, Trusler GA. Intraaortic balloon pumping in children. Ann Thorac Surg 1980;29:522-8.

13. Cassidy J, Haynes S, Kirk R, et al. Changing patterns of bridging to heart transplantation in children. $J$ Heart Lung Transplant 2009:28:249-54.

14. Hetzer R, Potapov EV, Stiller B, et al. Improvement in survival after mechanical circulatory support with pneumatic pulsatile ventricular assist devices in pediatric patients. Ann Thorac Surg 2006;82:917-24.

15. Potapov EV, Stiller B, Hetzer R. Ventricular assist devices in children: current achievements and future perspectives. Pediatr Transplant 2007;11:241-55.

16. Stiller B, Hetzer R, Weng Y, et al. Heart transplantation in children after mechanical circulatory support with pulsatile pneumatic assist device. J Heart Lung Transplant 2003;22: 1201-8.

17. Reinhartz O, Keith FM, El-Banayosy A, et al. Multicenter experience with the Thoratec ventricular assist device in children and adolescents. J Heart Lung Transplant 2001;20:439-48.

18. Rao V, Oz MC, Flannery MA, Catanese KA, Argenziano M, Naka Y. Revised screening scale to predict survival after insertion of a left ventricular assist device. $J$ Thorac Cardiovasc Surg 2003;125:855-62.

19. Gandhi SK, Grady RM, Huddleston CB, Balzer DT, Canter CE. Beyond Berlin: heart transplantation in the "untransplantable." J Thorac Cardiovasc Surg 2008;136:529-31.

20. Haddad H, Elabbassi W, Moustafa S, et al. Left ventricular assist devices as bridge to heart transplantation in congestive heart failure with pulmonary hypertension. ASAIO $J$ 2005;51:456-60.

21. Schuster M, Kocher A, John R, et al. B-cell activation and allosensitization after left ventricular assist device implantation is due to T-cell activation and CD40 ligand expression. Hum Immunol 2002;63:211-20. 